\title{
The Study Research on Indoor Positioning Technique
}

\author{
Zuo huan ${ }^{\mathrm{a}}$, Zhang na $^{*}$, Xu jian $^{\mathrm{b}}$, Shan Juan juan $^{\mathrm{c}}$, Jiao jian $^{\mathrm{d}}$ \\ College of Computer and Information Engineering, Beijing University of Agriculture, BeiJing, China \\ *Corresponding author: zhangna@bua.edu.cn \\ ${ }^{\mathrm{b}}$ bjnxy@126.com; ${ }^{\mathrm{c}} \mathrm{sjj} @$ bua.edu.cn
}

Keywords: Indoor Positioning; Technique; Category

\begin{abstract}
There has no one type of indoor positioning technique which can work in all scenarios. Because of the limitations of technical and the different concept of design, the existing technique of indoor positioning only applies to a particular scene. In this paper, the algorithms of indoor positioning algorithms and the comparison of familiar techniques will be discussed thoroughly, which will help the operators and researchers of indoor positioning technique to make a choice and research. Finally, the trends of the study of indoor positioning technique will be analyzed, and the drift of the difficulties and development of indoor positioning technique will be discussed as well.
\end{abstract}

\section{Introduction}

The prospect and research of spatial positioning is significant. Due to signal attenuation and multipath effects, GNSS (Global Positioning Satellite System) can't provide high accuracy and stable position information service for users indoor. Indoor positioning technique emerged as the focus of research experts and scholars, and began to develop rapidly. However, there is no one type of indoor positioning technique which can applies to every condition, due to limitations of technologies and different concepts the existing indoor positioning system are so design that it only work in a particular environment. Therefore, the indoor positioning technique are classified according to their characteristics, will help the operators and researchers of indoor positioning technique to make a choice and research.

\section{Indoor Location Algorithm}

GNSS(e.g. GPS) was widely used in the field of space orientation in the first place, range based techniques are extensively used in localization algorithm. Range based techniques are measured by wireless communication technique geometric parameters (such as measurement of distance or angle), and then use geometry space model to calculate the information of target's position by geometric principles. The main methods include: trilateral positioning method ${ }^{[1]}$, hyperbolic positioning method ${ }^{[2]}$ and triangulation method ${ }^{[3]}$. Of course, ensuing indoor positioning technique put to use such an approach, and achieved certain results. But the environment of indoor is complex which is different from outdoor, signal attenuation, multi-path effects and other factors affect the accuracy of the range based techniques of indoor positioning systems seriously. So comes range free indoor positioning method ${ }^{[4]}$ including scene analysis and proximity.

\subsection{Range Based Algorithm}

(1) Trilateral

On the plane, triangular location method have three reference points at least which are not on the same line. According to the distances to the three points estimated, combined with the position of the reference points, the position of the target is on the intersection of the three circles.

The method of ranging frequently-used for indoor positioning are Received Signal Strength Indication (RSSI) and Time Of Arrival (TOA). The source of the transmitted signal strength with increasing distance gradually weakened, the distance signal point and reception point determined by the strength of the received signal, known as RSSI ranging method. TOA is measured by the clock 
radio signal transmission time, thereby obtaining a distance signal point and the receiving point, but the signal source and the receiving point require clock synchronization which is difficult to achieve.

(2) Hyperbola

The geometric principles of hyperbola go like this: the difference of the two distances from point on hyperbola to two fixed points which are the focal points of the hyperbola is a constant. There reference points that are not on a straight line can be determined to two hyperbola, two hyperbola's intersection is the target point.

The method of ranging frequently-used for indoor positioning is Time Difference Of Arrival (TDOA) which avoid the requirement of clock synchronization, calculate the distance difference by figure out the difference of time-of-flight between the target point the two reference points.

(3) Angulation Techniques

Advantage of this fact: to a triangle, if known length of one side and the size of two angle connecting to the side, you can determine the position of the target which is on the intersection of the other two sides of the triangle.

In indoor positioning, the commonly algorithm used is Angle Of Arrival (AOA), which is detected by the base station receiver array antenna radio emission angle of incidence of the target, thereby forming a radially from the receiver to the mobile station connection, calculating angles.

\subsection{Range Free Algorithm}

(1) Scenes Analysis

In contrary to the parameter method, the method of scene analysis can effectively counter the impact of multipath propagation in the indoor, and can greatly improve the accuracy of indoor positioning. At present, the typical scene analysis indoor positioning technology is the location fingerprint positioning technology. Location fingerprint positioning technology is to use the access point of the RSS (Signal Strength Received) and the physical location of the correlation. That is to say, the use of RSS is not the same in different physical locations. RSS in different physical position of the performance by the position of the prior collection, extract the mapping relationship between the value of the location and the physical location of the training to build a fingerprint database, that is, the location of the fingerprint map. In the end, the real-time RSS is compared with the position fingerprint map and get the positioning results. Because of the intensity of the signal in the real environment, the intensity of the signal is random, and the static location fingerprint map can't reflect the actual situation in real time. Using the active reference label instead of the offline data acquisition, the dynamic reference information can capture the environmental changes more timely, and can improve the accuracy and credibility of the positioning.

(2) Proximity

Using the limited range of the signal, it can be determined whether the point is near to the reference point. But it can only provide approximate positioning information and meet the requirements of some applications.

\section{Indoor Positioning Technique}

The positioning algorithm described above, derived from a variety of indoor positioning technologies, including infrared, ultrasound, computer vision, Bluetooth, WiFi, RFID, ultra-wideband, and ZigBee.

\subsection{Mainstream indoor positioning technique}

(1) Infrared

Infrared (Ray Infrared) positioning of the infrared emitter which is emitted by the infrared emitter is received by an infrared emitter. AT\&T Cambridge laboratory developed the infrared outdoor positioning system Active Badge System ${ }^{[5]}$ is known as the first generation of indoor positioning system. Infrared indoor positioning accuracy is relatively high, but only within the visual range of linear transmission, by indoor layout and lighting effects, higher positioning cost.

(2) Ultrasonic

Ultrasonic positioning is a reflection method, based on the time difference between the transmitted ultrasonic wave to the reference node response echo and the distance between the 
reference nodes, the location of the target is calculated by triangulation. Active Bat ${ }^{[6]}$ is a pioneer of the ultrasonic positioning system, through the dense deployment of a large number of ultrasonic receiving equipment, to achieve the positioning accuracy of $3 \mathrm{~cm}$. The ultrasonic positioning accuracy is high, but the signal transmission attenuation is serious, the positioning range is limited, and the equipment cost is high.

(3) Computer Vision

The computer vision is an Easy Living system, which can accurately identify the moving target, extract the stable target features, and carry out the fast real-time tracking and calculation of the target's position. Computer vision indoor positioning accuracy is relatively high, but only on the visual range of the target, the effective distance is short, can't adapt to the complex environment.

(4) Bluetooth

Bluetooth indoor positioning based on the measurement of the signal intensity of the terminal equipment through the scene analysis algorithm. IBeacon is a protocol technology for the development of the Apple Corp, which is located in $2 \sim 3 \mathrm{~m}$. Bluetooth positioning technology has high security, low cost, low power consumption, small device size, the majority of mobile phone terminals are built-in Bluetooth module, easy to spread and deployment of large scale, but the technology is vulnerable to external noise signal interference, signal stability is poor, communication range is small.

(5) $\mathrm{WiFi}$

In recent years, the rapid development of WiFi technology, public places such as large supermarket shopping malls, schools, enterprises, etc. have been widely deployed WiFi. WiFi indoor positioning technology has been a lot of representative research results, such as RADAR system ${ }^{[7]}$ Nibble system ${ }^{[8]}$, Weyes system ${ }^{[9]}$ and other indoor positioning system. Because of the popularity of WiFi network, WiFi is a popular positioning technology, positioning accuracy can reach meter level, low positioning, positioning signal transmission range is large, and the applicability is good.

(6) RFID

The RFID(Radio Frequency Identification) positioning technology uses radio frequency signal to carry out the non - contact type two-way communication and exchange data to achieve the purpose of identification and location. At present, representative RFID positioning system has MIT Oxygen project development of Cricket system ${ }^{[10]}$, the SpotON system of University of Washington ${ }^{[11]}$, the Microsoft Corp of the RADAR system, University of Hong Kong Landmarc system etc.. RFID technology transfer range is large, the cost is very low, but the distance is short, the longest only a few tens of meters, and the radio frequency signal does not have the ability to communicate, but the use of radio frequency identification technology is not indoor positioning, must be combined with other auxiliary technology to complete.

(7) UWB

UWB (Ultra Wide Band) technology by sending data to the ultra-narrow pulse to transmit data, $\mathrm{GHz}$ level of data bandwidth, to achieve indoor positioning. Ubisense ${ }^{[12]}$ is a combination of TDOA and AOA indoor positioning system, ranging from $50 \mathrm{~m}$ to $15 \mathrm{~cm}$. UWB is difficult to achieve a wide range of indoor coverage, higher system construction costs have also limited the application of ultra wideband positioning technology.

(8) ZigBee

ZigBee ${ }^{[13]}$ indoor positioning is a kind of low power, low cost, short distance wireless network technology, commonly used in the field of industrial sensing and smart home.

\subsection{Comparison of indoor positioning technique}

Compare with the several current indoor positioning technology in several aspects of accuracy, positioning methods, advantages and disadvantages; from the macro grasp the direction of the development of indoor positioning technology and the current study of the bottleneck. 
Table 1 Comparison mainstream indoor positioning technique

\begin{tabular}{|c|c|c|c|c|}
\hline technique & Accuracy & Algorithm & advantage & disadvantage \\
\hline Infrared & $5 \sim 10 \mathrm{~m}$ & Proximity & High precision & $\begin{array}{l}\text { Can't cross barriers, high cost, } \\
\text { high power consumption by } \\
\text { light interference }\end{array}$ \\
\hline Ultrasonic & $1 \mathrm{~cm} \sim 10 \mathrm{~cm}$ & $\begin{array}{l}\text { Trilateral } \\
\text { Location }\end{array}$ & $\begin{array}{l}\text { Overall high } \\
\text { precision, simple } \\
\text { structure }\end{array}$ & $\begin{array}{l}\text { Multipath effects, attenuation } \\
\text { significantly affected by } \\
\text { temperature, high costs }\end{array}$ \\
\hline Visual & $1 \mathrm{~cm} \sim 1 \mathrm{~m}$ & Scene Analysis & $\begin{array}{l}\text { Environment-depend } \\
\text { ent low }\end{array}$ & High costs, low stability \\
\hline Bluetooth & $2 \mathrm{~m} \sim 10 \mathrm{~m}$ & $\begin{array}{l}\text { Proximity, } \\
\text { Trilateral }\end{array}$ & $\begin{array}{l}\text { Low power } \\
\text { consumption, easy } \\
\text { integration }\end{array}$ & $\begin{array}{l}\text { Short distance, poor stability, } \\
\text { susceptible to noise }\end{array}$ \\
\hline WiFi & $2 \mathrm{~m} \sim 50 \mathrm{~m}$ & $\begin{array}{l}\text { Scene analysis, } \\
\text { Proximity, } \\
\text { Trilateral }\end{array}$ & $\begin{array}{l}\text { Easy to install, high } \\
\text { overall system } \\
\text { accuracy }\end{array}$ & $\begin{array}{l}\text { Fingerprint collection } \\
\text { workload, be calibrated by } \\
\text { other signal interference, high } \\
\text { power consumption }\end{array}$ \\
\hline RFID & $5 \mathrm{~cm} \sim 5 \mathrm{~m}$ & Proximity & $\begin{array}{l}\text { High precision, low } \\
\text { cost, small size }\end{array}$ & $\begin{array}{l}\text { Short distance, no } \\
\text { communication capabilities to } \\
\text { identify, is not easy to } \\
\text { integrate }\end{array}$ \\
\hline UWB & $6 \mathrm{~cm} \sim 10 \mathrm{~cm}$ & Trilateral & $\begin{array}{l}\text { Penetrating power, } \\
\text { high accuracy, low } \\
\text { power consumption }\end{array}$ & High cost \\
\hline ZigBee & $1 \mathrm{~m} \sim 2 \mathrm{~m}$ & Proximity & Low power, low cost & $\begin{array}{l}\text { Low stability, subject to } \\
\text { environmental interference }\end{array}$ \\
\hline
\end{tabular}

\section{Research direction of indoor positioning technique}

From the analysis above, the indoor localization technological development at present mainly has the following several general character problem to need to be solved:

(1)Accuracy

At present, the locating technical is not accuracy enough, in several meters, this accuracy is more than in indoor environment, In order to increasing the accuracy and solving these traditional problems, we must enhance the anti-interference ability, solving the signal attenuation, multi-diameter effect, apparent distance propagation and signal shake calibration etc.

(2) Reduce consumption and cost problems

At present, most of the positioning technology need to install auxiliary nodes in the environment for ranging and return location information. In order to improve the accuracy, it is necessary to install a large number of auxiliary nodes, which greatly increases the cost and energy consumption. Some technologies need high power and cost much more energy consumption, some technology need a lot of manpower to collect fingerprint information, perfecting the fingerprint map. In addition, using RSSI ranging also need periodic artificial calibration. These problems have greatly restricted the development of the technology in terms of practicality. According to the construction of fingerprint map to improve the accuracy of WiFi positioning, the history of using mobile devices use location to improve fingerprint map, reduces the workload of artificial collecting fingerprints. Using the similarity of each floor structure in multistory buildings, an algorithm is proposed to relieve the workload of collecting fingerprints. There are some methods provides a self-calibration based on FM mechanism, overcome the error of RSSI ranging is shaken when the electromagnetic is waved. 


\section{(3) General standardized}

All kinds of positioning technologies have advantages and disadvantages, the future development trend of indoor positioning is the integration of multiple technologies, and learn from each other. But the standardization of system structure and the problem of the seamless integration of various technologies are restricting the trendy of development greatly. In addition, combine with the indoor positioning and the outdoor positioning is the trend of the times, as the same as it need to be involved in the standardization problem. So the improvement of the general standard is helpful to promote the development of indoor positioning technology. There isn't a unified authoritative standards promulgated of the current positioning technology, undoubtedly, it restricts the development of multi technology integration.

(4) Security problem

Part of the real-time location belong to personal privacy of individuals involved the safety problem. The current position information technology will be an object to be measured or calculated to return to the host database, but the host or database will be left at the end, then leaving some security risks. The purpose of the indoor guidance and monitoring system based on RFID is to protect user privacy, and not to disturb the user's daily life.

\section{Summary}

Indoor positioning technology is a hot research field and has a wide range of applications. The next step of research work can be carried out from the following aspects:

(1) Proposed new location methods. The current positioning technology is built in the vicinity of the information, scene analysis and geometric characteristics of three kinds of positioning methods, if you can put forward a new method, it is bound to be a new area of indoor positioning technology has played a huge role in promoting.

(2) Using new wireless media. The current positioning technology is based on the traditional wireless media, each has advantages and disadvantages. If we can put forward a new wireless medium, it can overcome the shortcomings, and will greatly promote the development of indoor positioning technology. At present, in addition to the introduction of several technologies, as well as FM, radar, GSM, etc., in addition, magnetic field, video recognition and positioning technology is also in the research phase.

(3) Multi technology integration. In view of the current mainstream technology have advantages and disadvantages, the fusion technology is a powerful method of each other. However, there are problems in the problem of universal standardization, the problem of the seamless connection of the technology, and the power consumption of the technology.

(4)Optimization of the existing technology. It can be seen from the comparison that the main shortcomings of the current technology are focused on the accuracy, the effort distance, vulnerable to interference, power consumption, the dependence on auxiliary facilities, the higher cost and the shortcomings of the former technology. That will greatly improve the practical application of the technology.

\section{References}

[1] Homayoun Hashemi, The Indoor Radio Propagation Channel. Proceeding of the IEEE, 1993, 81(7): 943 968.

[2] J. Ma, Q. Chen, D. Zhang. An Empirical Study of Radio Signal Strength in Sensor Networks. Technical Report, Dept. of Computer Science and Engineering, Hong Kong University of Science and Technology, March 25, 2006

[3] Yimin Zhang, Moeness G. Amin, Shashank Kaushik. Localization and Tracking of Passive RFID Tags Based on Direction Estimation. International Journal of Antennas and Propagation, 2007.

[4] Duda R O, Hart P E, Stork D G. pattern taxonomy - BeiJing: China Machine Press , 2003. 
[5] Want R,Hopper A,Falcao V,et al.The active badge location system.ACM Transactions on Information Systems(TOIS),1992,10(1):91-102.

[6] WardA, JonesA, HopperA. Anewlocation technique for the active office .Personal Communications,IEEE,1997,4(5):42-47.

[7] Bahl P,Padmanabhan V N.RADAR:An in-building RFbased user location and tracking system:INFOCOM 2000. Nineteenth Annual Joint Conference of the IEEE Computer and Communications Societies.Proceedings.IEEE.2000.Ieee.pp.775-784.

[8] Castro P,Chiu P,Kremenek T,et al.A probabilistic room location service for wireless networked environments: Ubicomp 2001:Ubiquitous Computing,2001.Springer.pp.18-34

[9] Kaemarungsi K,Krishnamurthy P.Modeling of indoor positioning systems based on location fingerprinting:INFOCOM 2004.Twenty-third AnnualJoint Conference of the IEEE Computer and Communications Societies,2004.IEEE.pp.1012-1022

[10] Smith A,Balakrishnan H,Goraczko M,et al.Tracking moving devices with the cricket location system: Proceedings of the 2nd international conference on Mobile systems.applications, and services,2004. ACM.pp.190-202.

[11] Hightower J,Want R,Borriello G.SpotON:An indoor 3D location sensing technology based on RF signal strength[R].UW CSE 00-02-02,University of Washington, Department of Computer Science and Engineering, Seattle,WA,2000,1.

[12] De Angelis A,Händel P,Rantakokko J.Measurement report.Laser total station campaign in KTH R1 for Ubisense system accuracy evaluation.KTH Royal Institute of Technology Tech. Rep.,Stockholm Sweden,2012.

[13] Jin M,Yu C,Lai H,et al.Zigbee positioning system for smart home application: Frontiers of High Performance Computing and Networking ISPA 2007 Workshops,2007. Springer.pp.183-192. 